\title{
Long standing exophytic conjunctival papilloma infected with human papillomavirus
}

\author{
Anvesh Annadanam¹, M. Adelita Vizcaino², Charles George Eberhart ${ }^{2}$, Gibran S. Khurshid ${ }^{3}$ and Praveena Gupta ${ }^{4 *}$ \\ Correspondence: prgupta@utmb.edu

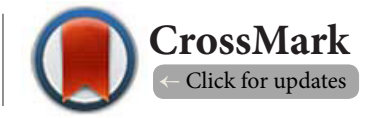 \\ 'Wilmer Eye Institute, Johns Hopkins University, Baltimore, MD, USA. \\ ${ }^{2}$ Department of Pathology, Johns Hopkins University, Baltimore, MD, USA. \\ ${ }^{3}$ Department of Ophthalmology, University of Florida, Gainesville, FL, USA. \\ ${ }^{4}$ Department of Ophthalmology and Visual Sciences, University of Texas Medical Branch, Galveston, TX, USA.
}

\begin{abstract}
Background: Most conjunctival papillomas are benign in nature. However, any long standing conjunctival mass should not evade the surveillance of carcinogenicity. Presented here is a case of a long standing conjunctival papilloma (HPV) that showed the presence of low risk human papillomavirus based on histological testing.

Methods: This is a retrospective review of a case of a patient with a longstanding conjunctival mass that was surgically excised. Baseline demographics, ocular history, and pathology reports are presented.

Results: A 48-year-old man presented for urgent care evaluation of a growth on his left lower eyelid that was itchy and bled intermittently with eye rub. Ocular examination of the lower eyelid revealed a large pink pedunculated mass on the palpebral conjunctiva. The patient underwent excision of the mass with conjunctivoplasty and ocular surface reconstruction. Histopathology of the tissue revealed the mass to be a conjunctival papilloma with positive staining for low-risk HPV types 6 and 11.
\end{abstract}

Conclusion: Although the chronic nature of the lesion reduces malignant potential, staining for low risk HPV 6 and 11 was present. Any longstanding papilloma warrants histopathology workup to help with appropriate medical or surgical management.

Keywords: Conjunctival papilloma, human papillomavirus, exophytic papilloma, conjunctival mass, conjunctiva

\section{Introduction}

Conjunctival papillomas are common in the eye [1] and fall into various categories including squamous cell, limbal, inverted, and viral. Prevalence in the United States ranges from 4 to $12 \%$, and formation is strongly associated with human papillomavirus (HPV) types 6 and 11 [2]. Here we present the case of a patient who had a conjunctival mass tucked under his left lower eyelid for 15 years. It was later diagnosed as an exophytic conjunctival papilloma that exhibited p16 staining and was found positive for HPV subtypes 6 and 11. The tumor was removed surgically without any complications.

\section{Case presentation}

A 48-year-old African American male presented to the urgent care clinic complaining "I have meat in my eye." Careful evaluation of the left lower eyelid revealed a large loose-hanging pink mass that was tucked inside the palpebral conjunctiva (Figure 1A). Per history, the mass had been present for the past 15 years, but had been increasing in size over the past couple of months (Figure 1B). The growth generally resides inside the eyelid but sometimes pops out and can be repositioned manually. Other than being a former smoker with hypertension and asthma, the patient did not have any significant ocular medical or surgical history. The patient was discharged with olopatadine drops to relieve any discomfort and was given an ambulatory referral to ophthalmology.

On ophthalmic examination, pupils were equal, round, and reactive to light, with no afferent pupillary defect bilaterally. Visual fields were full and extraocular movement was intact. The patient did not report any vision changes, and best cor- 
rected visual acuity was $20 / 20$ in both eyes. Slit lamp exam revealed normal cornea, anterior chamber, iris, lens, and fundus. Intraocular pressures for the right and left eyes were $26 \mathrm{~mm}$ and 23 millimeters of mercury, respectively. Slit lamp examination revealed a pedunculated papillomatous conjunctival mass of about $6 \times 12$ millimeters on the left lower palpebral conjunctiva. The mass depicted typical characteristics of a papilloma, displaying multiple lobules, cysts, and mucous deposits. The patient reported occasional bleeding, evidence of which was marked by scarring and redness (Figure 1C).

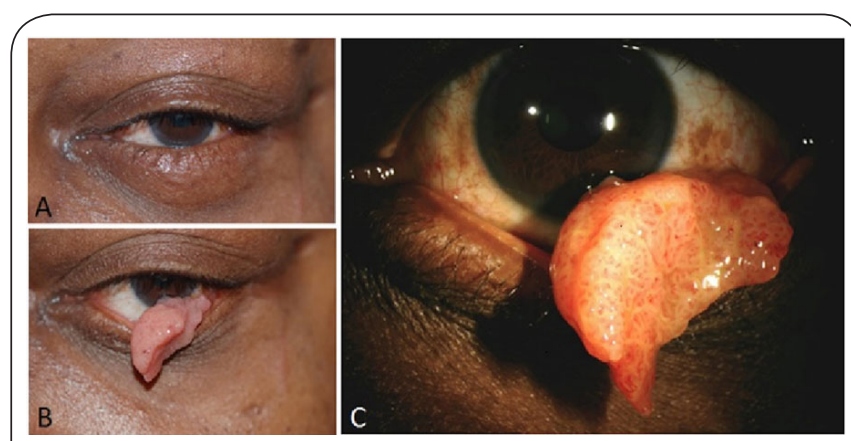

Figure 1. Gross images of lesion before excision:

[A] Fullness of eyelid, [B] Exophytic conjunctival papilloma,

[C] Multilobulated pedunculated conjunctival papilloma (high magnification).

The patient was referred to oculoplastics for surgical ex-cision of the lesion, conjunctivoplasty, and ocular surface reconstruction with amniograft. The mass was removed completely using iris scissors along with a small portion of the tarsus, to which it was partially adhered. The conjunctival defect was closed with interrupted suture. The specimen was sent to pathology for further characterization. The histopathology reportconfirmed the mass was a papilloma lined by conjunctival epithelium with numerous goblet cells (Figures $2 \mathrm{~A}$ and $2 \mathrm{2B}$ ). The lesion was inflamed with extensive neutrophils within the epithelium causing some reactive atypia, but no dysplasia was identified. Some koilocytic changes were noted, and nuclear p16 immunostains were positive in many cells (Figure $\mathbf{2 C}$ ). In situ hybridization revealed a few cells to be positive for low risk HPV 6/11 (Figure 2D). The patient had no complications from surgery and recovered well post operatively.

\section{Discussion}

Papillomas can be benign or malignant and can be found in many areas of the body. Specifically, conjunctival papillomas are common in the eye and are usually exophytic and rarely inverted [1]. Most papillomas have a central fibrovascular zone with multiple branching fronds that radiate from a base. Etiological factors include smoking, ultraviolet light, HPV infection, and immunodeficiency [2]. Pedunculated papillomas similar to the one reported here are strongly associated with infectious etiology. In the United States, prevalence of

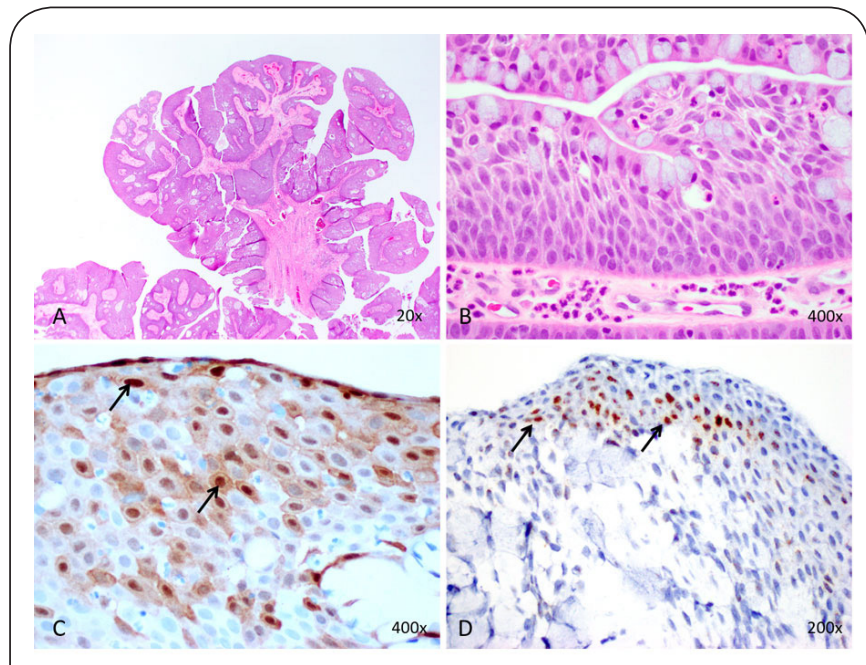

Figure 2. Pathology images after surgical excision of lesion: $[\mathbf{A}, \mathbf{B}]$ Hematoxylin and eosin stained sections show a large exophytic lesion consisting of branched fibrovascular cores lined by conjunctival epithelium with numerous goblet cells and intraepithelial neutrophils, $[\mathbf{C}]$ Many cells show nuclear p16 immunoexpression (arrows). [D] In situ hybridization for low-risk HPV 6/11 is also present in some cells (arrows).

conjunctival papilloma ranges from 4 to $12 \%$, and formation is strongly associated with HPV types 6 and 11 [3]. Although HPV 6 and 11 types are generally low risk, it can sometimes present with dysplasia. However, carcinoma rarely develops in conjunctival papilloma [4]. Squamous conjunctival papillomas, which are usually virally infectious, are found more commonly in children and young adults, and although generally not life threatening, they can become large in size and cosmetically displeasing. These tumors are associated with a maternal history of HPV infection, and may recur even after surgical excision [5]. Inverted conjunctival papillomas exhibit exophytic growth patterns and have many goblet cells interspersed within the epithelium. However, demonstration of dysplastic changes is uncommon. Most conjunctival papillomas are benign, but a case has been reported in which a conjunctival papilloma has extended to cause nasolacrimal duct obstruction [6]. On the other hand, the detection of HPV p16 through immunohistochemistry is alarming as they are usually associated with high risk uterine cervical intraepithelial neoplasia progressing to cervical cancer [4]. Based on the 2007 International research on cancer, conjunctival carcinogenicity of HPV in humans is limited [7].

Previous cases linking HPV and conjunctival papillomas have been published. Buggage et al., described a case of a 34-year-old HIV positive male with bilateral conjunctival lesions that were positive for HPV type 33 and recurred after surgically excised [8]. The high-risk nature of HPV type 33 and the patient's HIV status is likely to have contributed to recurrence, but we do not predict these to be of concern in our patient. Okan et al., presented the case of a five-year-old boy with HPV 
type 11 positive conjunctival lesions that recurred despite local excision and cryosurgery [9]. The lesion was described as nodular and irregular, in contrast to our patient's smooth, multilobulated lesion with well-defined borders.

The current case presented as a concern due to the lesion's cauliflower-like appearance, providing suspicion to the diagnosis of an infectious squamous cell papilloma. Although vision changes were not noted, the recent change in size of the mass provided a concern for malignancycaused by HPV infection. The longstanding nature of the mass reduces the risk of malignancy, however, any conjunctival lesion, even if present without problems for many years, should be sent for pathological evaluation to rule out the possibility of malignancy. Long-term proliferation may eventually lead to atypical and dysplastic changes. As such, it may have been safer if the patient had not waited for this long for evaluation of the mass. Our patient underwent full excision with conjunctival resurfacing surgery and an amniograft. The recurrence of viral papillomas is not uncommon, and completely excised papillomas do not generally recur. Given that our patient's lesion was not malignant, we predict a low chance of recurrence.

\section{Conclusions}

Gross appearance of conjunctival lesions may indicate malignancy. Surgical biopsy and histopathological workup of conjunctival lesions is warranted regardless of longstanding nature to help plan for medical treatment.

\section{Competing interests}

The authors declare that they have no competing interests.

\section{Authors' contributions}

\begin{tabular}{|l|c|c|c|c|c|}
\hline Authors' contributions & AA & MAV & CGE & GSK & PG \\
\hline Research concept and design & -- & -- & -- & -- & $\checkmark$ \\
\hline Collection and/or assembly of data & $\checkmark$ & -- & -- & -- & $\checkmark$ \\
\hline Data analysis and interpretation & $\checkmark$ & $\checkmark$ & $\checkmark$ & $\checkmark$ & $\checkmark$ \\
\hline Writing the article & $\checkmark$ & -- & -- & -- & $\checkmark$ \\
\hline Critical revision of the article & $\checkmark$ & $\checkmark$ & -- & -- & $\checkmark$ \\
\hline Final approval of article & $\checkmark$ & -- & -- & -- & -- \\
\hline Statistical analysis & -- & -- & -- & -- & -- \\
\hline
\end{tabular}

\section{Acknowledgement}

We would like to thank the photography department of the Wilmer Eye Institute at the Johns Hopkins Hospital for providing clear and timely images.

\section{Publication history}

Editor: Carsten H. Meyer, Pallas Clinic, Switzerland. Received: 09-Jan-2017 Final Revised: 11-Feb-2017 Accepted: 20-Feb-2017 Published: 06-Mar-2017

\section{References}

1. Grossniklaus HE, Green WR, Luckenbach and Chan CC. Conjunctival lesions in adults and histological review. Cornea. 1987; 6:78-116.

2. Kalantzis G, Papaconstantinou D, Georgalas I, Tsitlidou A and Georgopoulos G. Different types of conjunctival papilloma presenting in the same eye. Orbit. 2010; 29:266-8. | Article | PubMed
3. Sjo NC, von Buchwald C, Cassonnet P, Norrild B, Prause JU, Vinding T and Heegaard $S$. Human papillomavirus in normal conjunctival tissue and in conjunctival papilloma: types and frequencies in a large series. $\mathrm{Br} J$ Ophthalmol. 2007; 91:1014-5. | Article | PubMed Abstract | PubMed FullText

4. C DK and M MM. Advances in Diagnosis and Treatment of HPV Ocular Surface Infections. Med Hypothesis Discov Innov Ophthalmol. 2015; 4:31-5. | PubMed Abstract | PubMed FullText

5. Minchiotti S, Masucci L, Serapiao Dos Santos M, Perrella E, Graffeo $\mathrm{R}$, Lambiase $A$ and Bonini S. Conjunctival papilloma and human papillomavirus: identification of HPV types by PCR. Eur J Ophthalmol. 2006; 16:473-7. I Pdf I PubMed

6. Lauer SA. Recurrent conjunctival papilloma causing nasolacrimal duct obstruction. Am J Ophthalmol. 1990; 110:580-1. | PubMed

7. Ateenyi-Agaba $C$, Franceschi S, Wabwire-Mangen F, Arslan A, Othieno E, Binta-Kahwa J, van Doorn LJ, Kleter B, Quint W and Weiderpass E. Human papillomavirus infection and squamous cell carcinoma of the conjunctiva. Br J Cancer. 2010; 102:262-7. | Article | PubMed Abstract | PubMed FullText

8. Buggage RR, Smith JA, Shen D and Chan CC. Conjunctival papillomas caused by human papillomavirus type 33. Arch Ophthalmol. 2002; 120:202-4. | Article | PubMed

9. Okan G, Ayan I, Karslioglu S, Altiok E, Yenmis G and Vural G. Conjunctival papilloma caused by human papillomavirus type 11 treated with systemic interferon in a five-year-old boy. Turk J Pediatr. 2010; 52:97100. I PubMed

\section{Citation:}

Annadanam A, Vizcaino MA, Eberhart CG, Khurshid GS and Gupta P. Long standing exophytic conjunctival papilloma infected with human papillomavirus.

J Eye Ophthalmol. 2017; 4:1.

http://dx.doi.org/10.7243/2055-2408-4-1 